九州大学学術情報リポジトリ

Kyushu University Institutional Repository

\title{
Analysis of crossing collision accident characteristics by accident party
}

Dong, Jiaao

Department of Automotive Science, Graduate School of Integrated Frontier Sciences, Kyushu University

Hirota, Masaki

Department of Automotive Science, Graduate School of Integrated Frontier Sciences, Kyushu University

Nomura, Takumi

Department of Automotive Science, Graduate School of Integrated Frontier Sciences, Kyushu University

Sato, Junya

Department of Automotive Science, Graduate School of Integrated Frontier Sciences, Kyushu University

http://hdl. handle. net/2324/4102494

出版情報: International Journal of Intelligent Transportation Systems Research, pp.1-, 202011-02. Springer Science+Business Media

バージョン :

権利関係 : 


\title{
Analysis of crossing collision accident characteristics by accident party
}

\author{
Jiaao Dong \& Masaki Hirota * \& Takumi Nomura \& Junya Sato \\ Department of Automotive Science, Graduate School of Integrated Frontier Sciences, \\ Kyushu University \\ (744 Motoka Nishi Ward Fukuoka Japan, hirota.masaki.972@m.kyushu-u.ac.jp)
}

\begin{abstract}
This paper shows the characteristics of crossing collision accident by accident party. As Fukuoka Prefecture has the third most accidents in Japan, we selected Hakata and Chuo Ward in Fukuoka city as the target of the investigation. We investigated 1,810 crossing collision accidents and 2,662 intersections' environmental factors such as traffic lights and intersection shape. In addition, we classified the accidents into car to car accidents and car to bicycle accidents according to the type of accident parties involved. Characteristics of car to car accidents tending to occur at crossroads and car to bicycle accidents occurring regardless of the traffic regulations have been clarified.
\end{abstract}

Keywords accident, crossing collision, accident party, environmental factor

\section{Introduction}

The number of traffic accidents in Japan in 2018 was recorded to be $430,601^{[1]}$. The types of accidents were classified as vehicle-to-vehicle accident, person-tovehicle accident, vehicle alone accident, or train accident ${ }^{[2]}$. Amongst these, $86.1 \%$ were vehicle-to-vehicle accidents accounting for the most ${ }^{[3]}$. In vehicle-to-vehicle accidents, the most common are rear-end collisions $(40.3 \%)$, and the second most common are crossing collision accidents $(28.8 \%)^{[3][4]}$.

In recent years, due to the use of collision damage reduction braking control devices by ADAS [5], the number of rear-end collision accidents is expected to decrease in the future. On the other hand, the crossing collision is an accident in which vehicles progressing in opposite directions collide with each other ${ }^{[6]}$. New measures are required as the driving support systems recently being used are not effective.

In previous research, the characteristics of crossing collision accident were clarified by analyzing the effect of intersection configurations based on the actual accident data. Sato et al. conducted a characteristic analysis of crossing collision accidents based on the intersection environment and accident information such as the intersection shape and traffic signal type ${ }^{[7][8]}$. Nomura et al. found elements that Sato et al. have not shown a relationship with the crossing collision accident by using the mutual influence analysis method for the intersection constituents. He showed the possibility that one measure would lead to the suppression of accidents at multiple intersections ${ }^{[9]}{ }^{[10]}$. ITARDA also analyzed the factors of the accident from the viewpoints of the road environment and human factors, clarified the actual conditions of the crossing collision accident [11] [12]. However, there is no research report on accident feature analysis which is classified by accident party involved using actual accident data. Especially when one of the parties involved in the accident is a bicycle. Bicycles do not require a license and run on sidewalks in Japan, so it is considered that the characteristics affected by the intersection environment are different from the characteristics affected by the accident between cars or motorcycles. Hagita et al. discovered the characteristics of bicycle accidents using actual information such as the direction of traffic in the actual accident ${ }^{[13]}{ }^{[14]}{ }^{[15]}$. In addition, Suzuki et al. also used actual bicycle accident information to discover the characteristics of bicycle accidents in urban areas ${ }^{[16]}[17]$. However, their analysis did not specially point out the characteristics of bicycle crossing collision accidents, nor did it compare with the characteristics of ordinary cars or motorcycles. In order to prevent both bicycle-related crossing collision accidents and car or motorcycles' accidents, it is necessary to separate the parties involved in each accident and use actual accident data to compare the frequency of accidents and environmental information at intersections.

The number of traffic accidents in Fukuoka prefecture in 2017 were 34,862, the third highest in Japan ${ }^{[18]}$. Amongst the prefectures in Fukuoka, Hakata Ward and Chuo Ward, which are in Fukuoka City, had 717 crossing collision accidents, accounting for $31.2 \%$ of the crossing collision accidents that occurred in Fukuoka City ${ }^{[19]}$. Therefore, we investigated the occurrence of accidents in Hakata Ward and Chuo Ward based on the accident data from 2015 to 2017, provided by the Fukuoka Prefectural Police. In addition, the intersections where the accident was encountered, was also investigated for the environmental factors of the intersections.

In this paper, we classify crossing collision accidents into car to car and car to bicycle according to the parties involved in the accident. Based on the environmental factors of the intersection where the actual accident occurred, the characteristics of car to car crossing collision accident and the car to bicycle crossing collision accident were clarified. 


\section{Analysis Methods}

\section{2-1. Classification method for crossing collision accidents by parties involved}

We obtained accident information from the Fukuoka Prefectural Police on the accidents that occurred in Hakata and Chuo Wards of Fukuoka City in Fukuoka Prefecture from 2015 to 2017. The accident information includes the location (latitude and longitude) of the accident, the shape of the road, the type of the first or second party of the accident, gender age of the party, degree of damage to the party, weather, violation of the law of the parties, human factors and environment ${ }^{[20]}$.

According to the National Police Agency statistics, the crossing collision accidents between vehicles encountered all over Japan in 2018 was about $85.1 \%$ of crossing collision accidents that occurred at intersections ${ }^{[1]}$. Since there are many crossing collision accidents at intersections, and it was necessary to clarify their characteristics. Therefore, we gathered the data of 1,810 accidents for analysis in this paper that occurred at the intersections in Hakata Ward and Chuo Ward, Fukuoka city.

\section{2-2. Intersection survey}

We surveyed the 1,036 intersections where the crossing collision accidents were encountered and 1,626 intersections, that were Accident-free intersections between Hakata and Chuo Ward, in Fukuoka City from


environmental factors related to the accidents at these intersections.

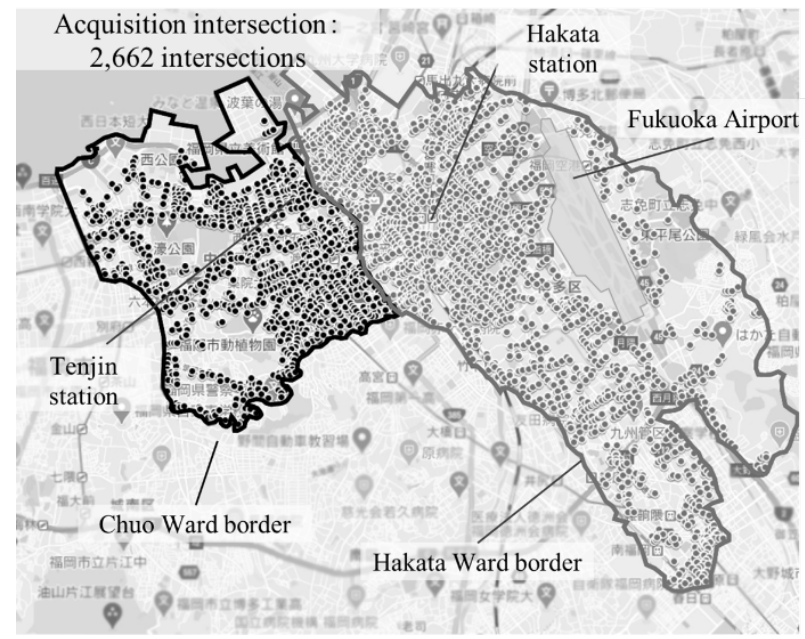

Fig.1 Intersections in Hakata Ward and Chuo Ward surveyed

Environmental factors of the intersection were confirmed from the image by satellite view and street view module through Google Map ${ }^{[21]}$. 2,662 intersections were examined for the shape of the intersection, the type of traffic signal, the road traffic regulation, the stop sign regulation, the stop sign setback. (Fig.2)

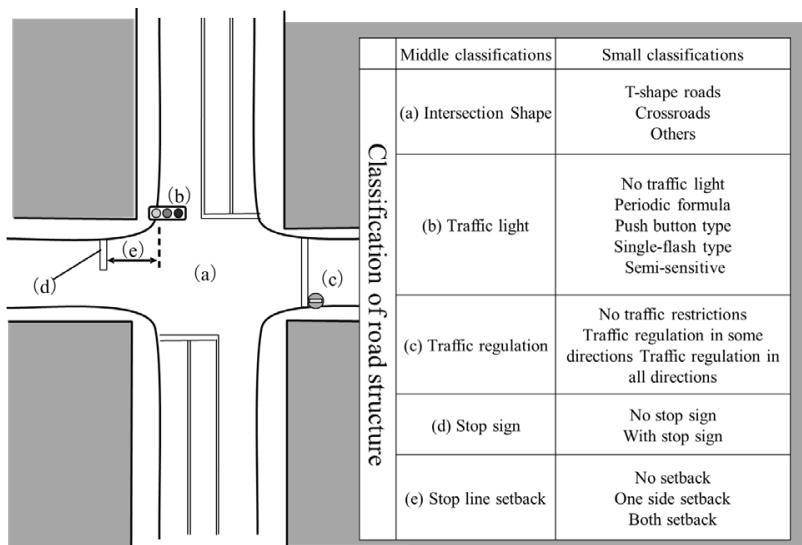

Fig.2 Constituent elements of the intersection surveyed

\section{2-3. Classification method for crossing collision accidents by parties involved}

The type of first party or the second party of the accident is described in the crossing collision accident information received from the Fukuoka Prefectural Police [20], which is used to classify the crossing collision accident into car to car accident and car to bicycle accident (Fig 3).

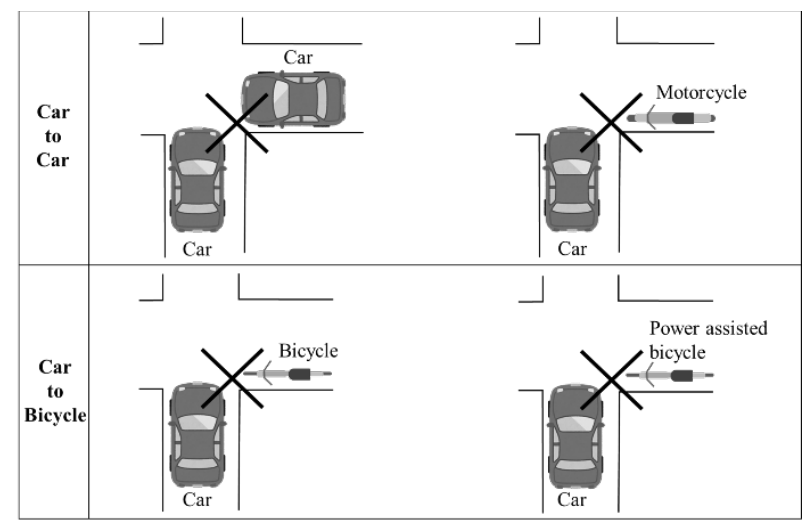

Fig.3 Accident type of crossing collision accident by party

Car to car accident is when both the first and second parties involved in the accident are cars. The reason why motorcycles are also classified into the category of cars is that while driving a motorcycle in Japan, a motorcycle license is required in the same way as ordinary cars, and the driver who is riding the motorcycle was also taught the same set of rules as that for the driver of a car. Therefore, while analyzing the characteristics of each party, the characteristics were like that of a car when compared to a bicycle that does not require a license.

On the other hand, car to bicycle accident is an accident in which a bicycle is involved in either the first party or the second party of the accident. Bicycles refer to normal bicycles and bicycles with power assist. 
Table.1 Determination of $\mathrm{i}$ and $\mathrm{j}$ depending upon Accident type by party and classification of intersection structure

\begin{tabular}{|c|c|c|c|c|c|c|c|c|c|c|c|c|c|c|c|c|c|c|}
\hline \multirow{3}{*}{$\begin{array}{c}\begin{array}{c}\text { Middle } \\
\text { classifications }\end{array} \\
\\
\text { Small } \\
\text { classifications }\end{array}$} & \multicolumn{2}{|c|}{ Accident type by party } & \multicolumn{16}{|c|}{ Classifications of intersection structure } \\
\hline & & & \multicolumn{3}{|c|}{ (a) Intersection Shape } & \multicolumn{5}{|c|}{ (b) Traffic light } & \multicolumn{3}{|c|}{ (c) Traffic regulation } & \multicolumn{2}{|c|}{ (d) Stop sign } & \multicolumn{3}{|c|}{ (e) Stop line setback } \\
\hline & $\begin{array}{l}\text { } \\
\delta \\
0 \\
\AA\end{array}$ & 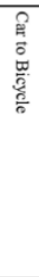 & 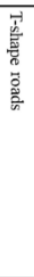 & 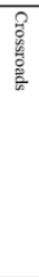 & 윰 & 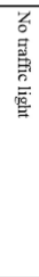 &  & 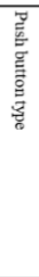 & 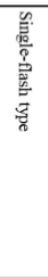 & 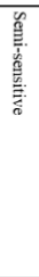 & 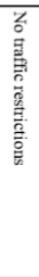 & 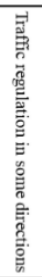 &  & 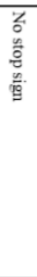 & 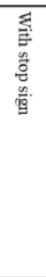 & 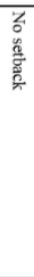 & 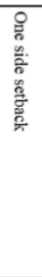 & 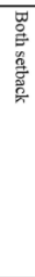 \\
\hline$i$ & 1 & 2 & & & & & & & & & & & & & & & & \\
\hline$j$ & & & 1 & 2 & 3 & 4 & 5 & 6 & 7 & 8 & 9 & 10 & 11 & 12 & 13 & 14 & 15 & 16 \\
\hline
\end{tabular}

In addition, another type of accident is a non-target party accident. Non-target party accident is accident in which either the first party or the second party of the accident could not be identified due to an unknown cause. Only a small number of accidents of this type were encountered $(0.77 \%$ of all accidents). Hence, they are excluded from the analysis in this paper.

\section{2-4. Evaluation method of accident frequency by intersection environmental factors}

From the intersection environmental factors surveyed in Hakata Ward and Chuo Ward, the number of accidents at each intersection and the rate of accidents per year (accident rate $\mathrm{R}_{\mathrm{ij}}$ ) was calculated.

$$
\mathrm{R}_{i j}=\frac{\mathrm{x}_{i j}}{3 \mathrm{~N}_{j}} \quad(\mathrm{i}=1,2, \mathrm{j}=1,2 \ldots 16) \cdots \cdots(1)
$$

$\mathrm{X}_{\mathrm{ij}}$ in equation (1) represents the number of accidents that occurred in the period of three years at the intersection that has ' $i$ ' type of the accident parties involved, ' $j$ ' element is the classification of intersection structure, and $\mathrm{N}_{\mathrm{j}}$ represents the number of intersections with ' $\mathrm{j}$ ' being the element of the classification of intersection structure.

' $\mathrm{i}$ ' is divided into car to car accident $(\mathrm{i}=1)$ and car to bicycle accident $(i=2)$, as shown in Table.1. ' $j$ ' is the environmental element of the surveyed intersections in Hakata and Chuo Wards. The reason for dividing the accident rate by 3 is that the accident information is received from the Fukuoka Police Department for a period of 3 years.

Taking equation (2) as an example, if we want to calculate the accident rate of car to bicycle accident at $\mathrm{T}$ shape roads, the accident rate of car to bicycle accident (i $=2)$ at $\mathrm{T}$-shape roads $(\mathrm{j}=1)$ is expressed as $\mathrm{R}_{21}$. While calculating, 396 car to bicycle accidents occurred at Tshape roads for a period of 3 years. Therefore, $\mathrm{x}_{21}$ is valued as 396. On the other hand, the number of T-shape road intersections surveyed in Hakata and Chuo Ward are 1,360. Therefore, $N_{1}$ is 1,360 . According to the formula (1),

$$
\mathrm{R}_{21}=\frac{\mathrm{x}_{21}}{3 \mathrm{~N}_{1}}=\frac{396}{3 \times 1360}=0.10
$$

The accident rate is 0.10 as shown in equation (2). By combining each element of Table.1, calculating sequentially, and comparing the accident rates of car to car accident and car to bicycle accident in different intersection environments, the characteristics of crossing collision accidents by parties will be clarified.

\section{Analysis Result}

\section{3-1. Analysis Result by shape of intersection}

This part clarified the characteristics of the accident by party, by accident rate comparison among different shapes of the intersection. The intersections of Hakata Ward and Chuo Ward are divided into T-shape intersections, crossroads, and others according to the shape of the intersection. Category named 'others' in Fig.4 refers to intersections where there are more than 4 crossing roads intersecting at a point.

Fig.4 shows the distribution of number of intersections by characteristics of intersection shapes. Amongst 2,662 intersections surveyed, T-shape roads were the most common type of intersection, accounting for half of the total intersections. On the other hand, there are few intersections of 'others' type in about 51 places.

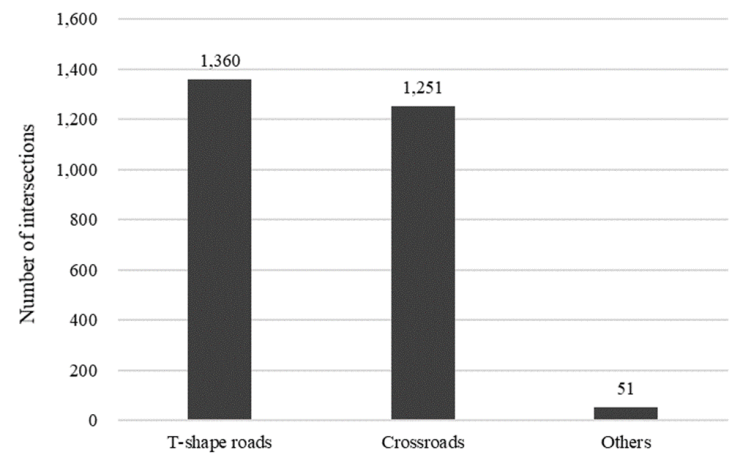

Fig.4 Number of intersections by intersection shape 
The distribution of the number of accidents occurring at intersections of each shape is shown in Fig. 5 by parties involved. Both car to car accidents and car to bicycle accidents are more at crossroads. However, compared to a car to car accident, there were about 100 fewer car to bicycle accidents at crossroads and about 220 more at Tshape roads.

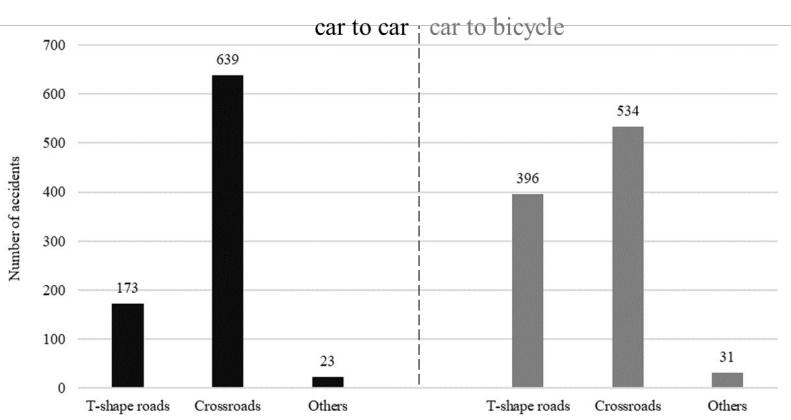

Fig. 5 Number of accidents by intersection shape (by party)

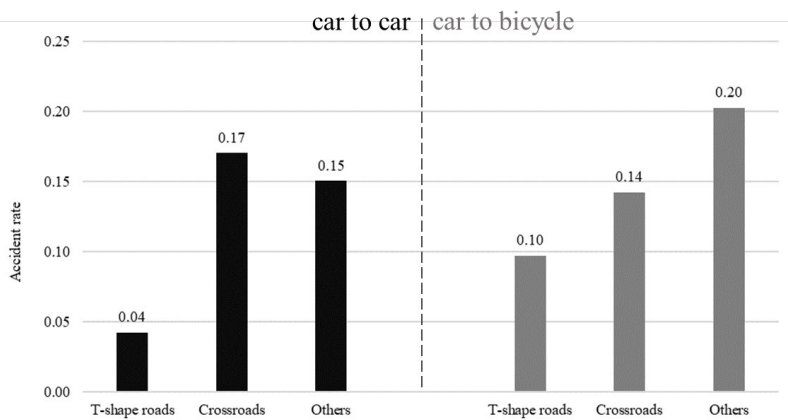

Fig.6 Accident rate by intersection shape (by party)

The distribution of accident frequency by party is shown in Fig.6 by calculation. As a result, it was found that car to car accidents were more likely to occur at crossroads and other specially shaped roads. On the other hand, it was found that car to bicycle accidents tend to occur due to an increase in the number of intersection roads or an increase in road complexity.
Therefore, we considered the cause of this difference. Compared to cars, bicycles can not only ride on the roads but also on sidewalks, so when the crossing roads increase, the activity range of bicycle increases more than cars. With increasing range, the collision points between car and bicycle also increases, which may lead to more crossing collision accidents.

\section{3-2. Analysis result by traffic light}

This part clarified the characteristics of the accidents by party, by accident rate comparison among different type of traffic signals at the intersection. The traffic lights at the intersections are classified into no traffic light, periodic formula, push button type, single-flash type, and semi-sensitive traffic signals.

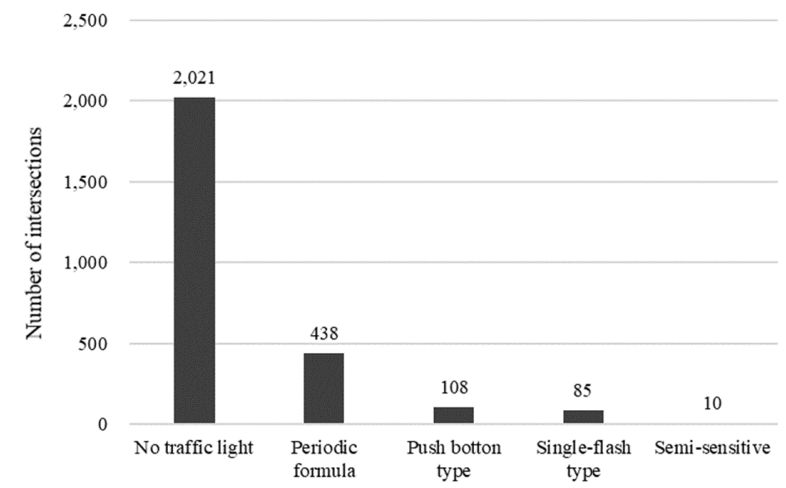

Fig.7 Number of intersections by Traffic light

Fig.7 shows the distribution of the number of intersections depending on the type of traffic light at the above intersections. Of all the intersections, there are 2,021 intersections with No traffic lights, which is the largest in number. Except for 438 places where the periodic formula is installed, there are few remaining three types of traffic lights.

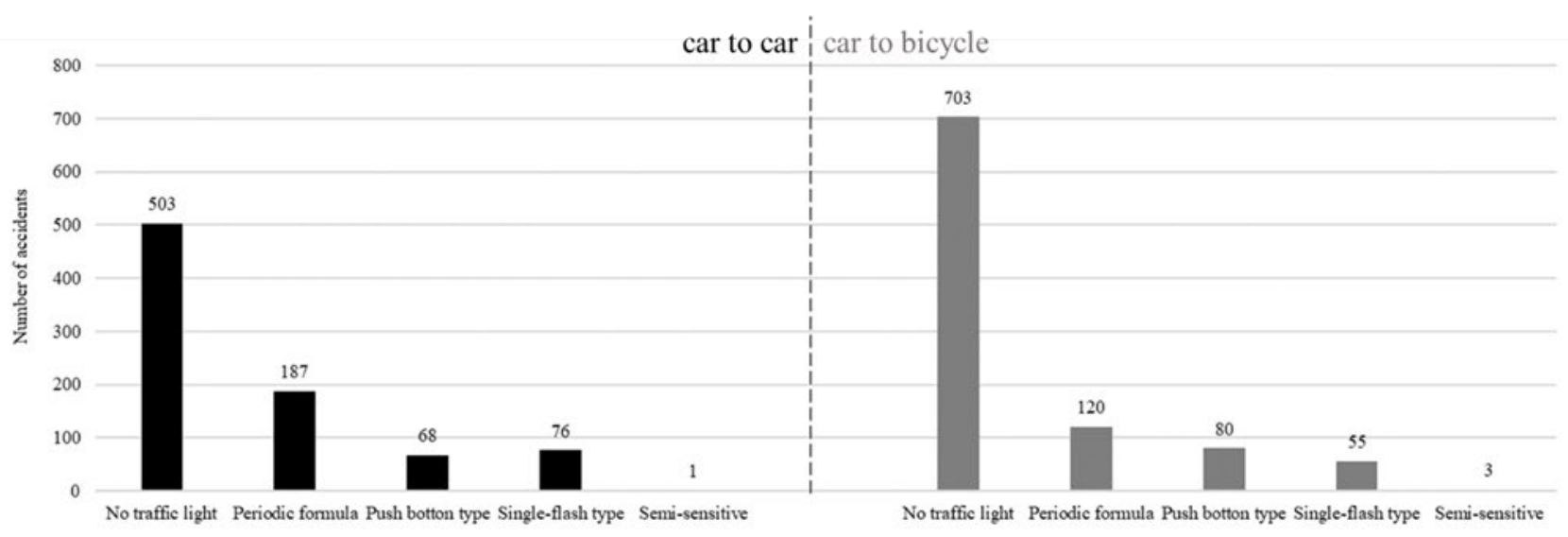

Fig.8 Number of accidents by type of traffic light (by party) 


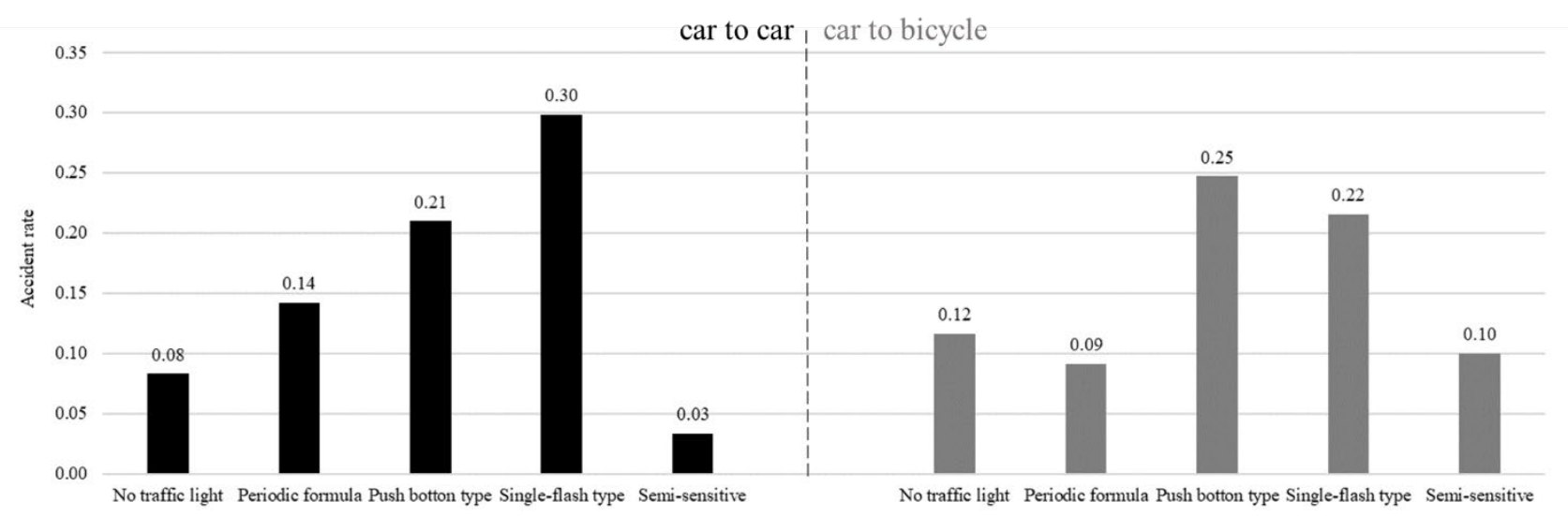

Fig.9 Accident rate by traffic light (by party)

The distribution of the number of accidents that occurred at intersections where the above-mentioned five types of traffic lights were installed by party is showed in Fig.8. Both car to car accidents and car to bicycle accidents occur at the intersections with no traffic light. However, there were 200 more car to bicycle accidents than car to car accidents occurred. In addition, the signal type of periodic formula, which is the second highest in number of intersections, the number of car to bicycle accidents is less than car to car accidents.

By using the equation (1) that summarizes the number of accidents and the number of intersections, the accident rate is calculated, and Fig.9 shows the distribution of accident frequency for each party. Comparing the features by party, car to car accidents are more likely to occur at the single-flash type or push button type intersection, and 1.75 times more likely occurred at the periodic formula intersections than no traffic light intersections. On the other hand, car to bicycle accidents are also more likely to occur with the single-flash type or push button type, but unlike car to car accidents, it is 1.33 times more likely to occur at no traffic light intersections than periodic formula intersections.

Therefore, it can be assumed that different causes of accidents incur in cars and bicycles due to difference in the frequency of accidents between parties in periodic formula and no traffic light. Due to the presence of periodic formula traffic signal, the driver tends to reduce the safety check like looking out for vehicle on the other intersecting road. Similarly, if the second party also tends to do the same due to presence of periodic formula traffic signal, then the two parties are more likely to collide with each other. On the other hand, unlike drivers of car, some of bicycle riders do not receive education on road signs, etc., so they tend to not follow the regulation of stopping before crossing at No traffic light intersections. According to ITARDA analysis, of the 200 car to bicycle accidents, the bicycle`s side was given priority as low as about $10 \%$, and it was found that there were many mistakes on the bicycle side ${ }^{[22]}$. Due to this reason, it is possible that bicycle may suddenly appear at an intersection without temporarily stopping at the stop line and meet with an accident.

\section{3-3. Analysis result by traffic regulation}

This part clarified the characteristics of accidents by party, by accident rate comparison among different types of traffic regulation at intersections. If the intersections are classified according to the traffic regulation of the crossing roads at the intersections, they can be classified into three types: No traffic regulations, traffic regulation in some directions and traffic regulation in all directions. 'No traffic regulations' intersection is an intersection where there is no one-way restriction on any intersection road, Traffic regulation in some directions is an intersection where there are some one-way restrictions but not at all intersecting roads. And traffic regulation in all directions are the intersections where all intersecting roads are with one-way restrictions.

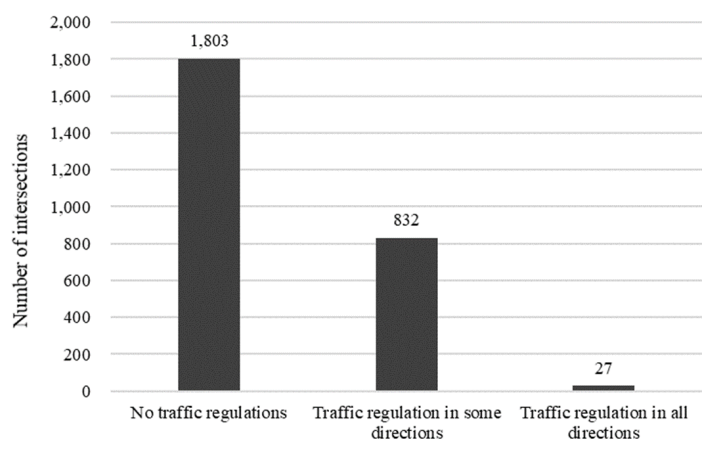

Fig.10 Number of intersections by traffic regulation

The distribution of the number of intersections classified according to the characteristics of these traffic regulations is showed in Fig.10. Of all the intersections, there are 1,803 intersections with no traffic restrictions, which are the highest in number. Intersections with traffic regulation in some directions are in 832 places, which is the second highest in number. There are only 27 intersections with traffic regulation in all directions. 


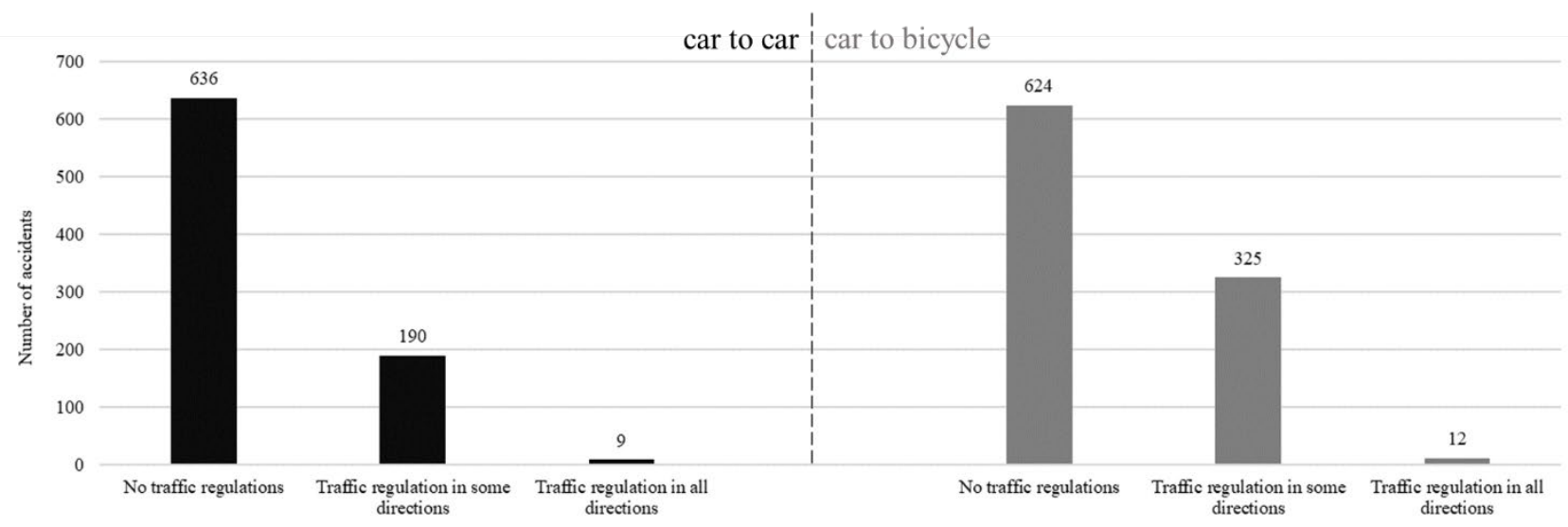

Fig.11 Number of accidents by traffic regulation (by party)

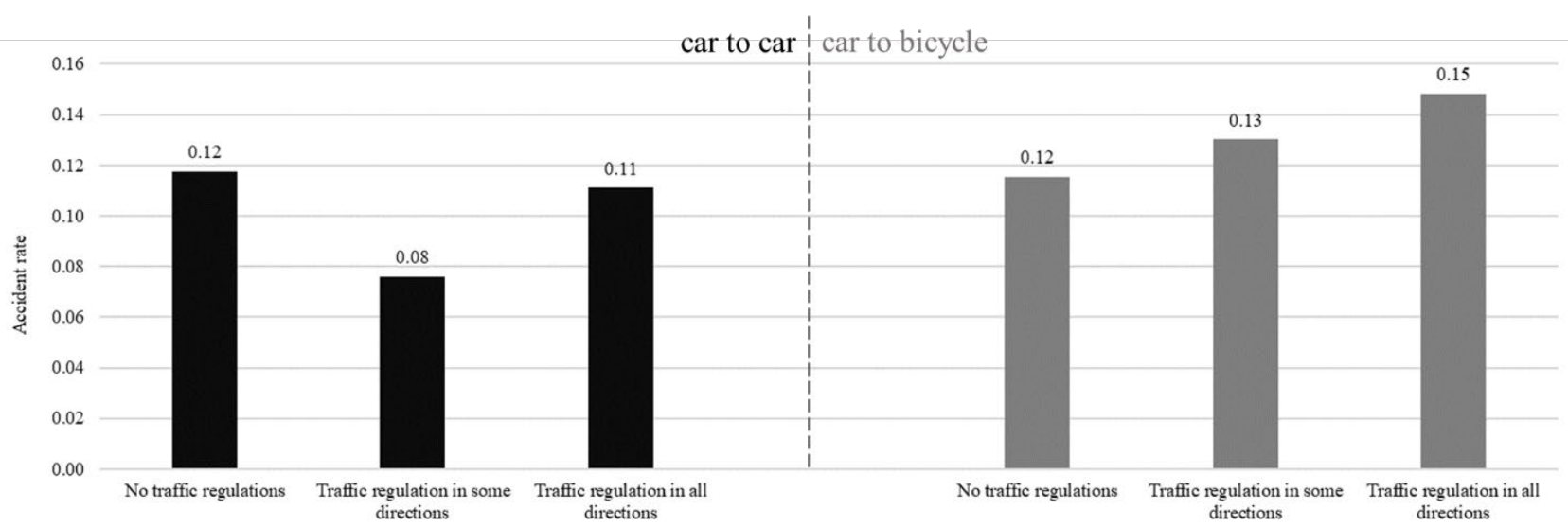

Fig.12 Accident rate by traffic regulation (by party)

Fig.11 shows the distribution of the number of accidents by party that occurred at intersections subject to the three types of traffic regulation. Both car to car accidents and car to bicycle accidents occur at intersections with no traffic regulations, and the number of accidents is almost the same. However, in traffic regulation in some directions, car to bicycle accidents occurred 135 times more than car to car accidents.

Calculating the accident rates, Fig.12 shows the distribution of accident frequency by party. Comparing the characteristics by party, the accident rate of car to car accidents tends to be reduced by $30 \%$ due to the direction regulation of some intersection roads. On the other hand, it was found that the car to bicycle accidents occur regardless of the direction regulation at the intersection.

Therefore, we considered the cause of this difference. According to the traffic regulation standards published by the National Police Agency, one of the characteristics of roads subject to traffic regulation is that, the road width is not enough for the vehicles to pass each other and it is necessary to ensure safety and flow of traffic ${ }^{[23]}$. In other words, traffic regulated roads are characterized by width of the road. The reason why the car to car accident rate decreases on traffic regulated roads is that the speed reduction is more effective than ordinary roads because the width of the road is reduced.


Fig.13 Distribution of road by lanes due to traffic restrictions

In response to this scenario, we conducted a survey as shown in Fig.13. We took 3,993 roads in the intersections between Hakata and Chuo Ward as the analysis object, divided them into roads with traffic regulation and roads with no traffic regulation, and classified them by the number of lanes. It can be seen from the figure that the ratio of 1 lane is $17 \%$ higher than that of no traffic regulation roads with traffic regulation, and roads with more than 2 lanes are almost non-existent. The lesser the number of lanes, the narrower the road, which also directly affects the speed of passing vehicles. This lowered speed at narrow roads may be the reason for the lower accident rates at narrow roads. As for the reason why the accident rate of traffic regulation on all directions 
has risen again, is because we can see that there are only 9 accidents samples in this part of the data in Fig.11, which may have lost its meaningful value compared to other data.

On the other hand, the reason why a car to bicycle accident occurs regardless of the direction regulation is because lots of riders of bicycle may not know the bicycle is considered as a light vehicle in Japan because they do not require a license to ride a bicycle as mentioned previously. They cannot understand the meaning of oneway traffic due to this cause, and we can assume that an accident will occur in reverse running by bicycle.

\section{3-4. Analysis result by stop sign}

\section{3-4-1. By Presence or absence of stop line}

This part clarified the characteristics of the accidents by party, by accident rate comparison with or without a stop sign at the intersection. According to the presence or absence of a stop sign on the intersection road, the surveyed intersections are classified into two types: no stop sign and with stop sign.

However, there is one limitation, because the stop sign regulation is where a vehicle must temporarily stop at the intersections, due to road signs in their vicinity etc. excludes this analysis. Therefore, the target intersections are 2,227 intersections except the intersection of periodic formula. At these 2,227 intersections, We confirmed whether there are stop signs at these intersections. Amongst them, No stop sign means that there is no stop sign in any cross road at the intersection. On the other hand, With stop sign refers to the presence of stop sign on any roads at the intersection.

Fig.14 shows the distribution of the number of intersections classified according to the characteristics of these stop signs. Out of 2,227 intersections, there are 1,610 places with stop sign, accounting for about $70 \%$ of the intersections. On the other hand, there are 617 intersections with no stop signs, accounting for about $30 \%$ of the intersections.

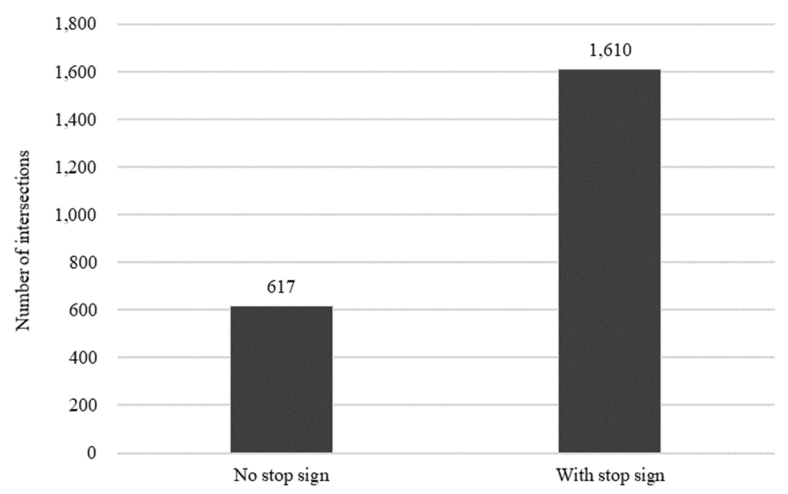

Fig.14 Number of intersections by stop sign
The number of accidents that occurred at the intersections with and without the above two types of stop signs by party is showed in Fig.15. Both car to car and car to bicycle accidents occur at many intersections with stop sign, the number of accidents occurred at with stop sign intersections is higher than that occurred on no stop sign intersections.

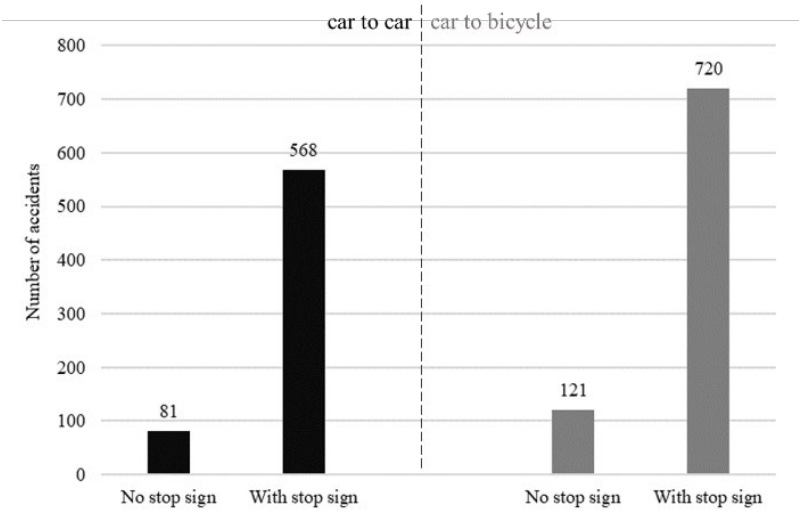

Fig.15 Number of accidents by stop sign (by party)

Furthermore, we calculated the accident rate in Fig.16. Car to car accidents are three times more likely to occur at intersections with stop sign than at intersections without stop sign. On the other hand, car to bicycle accidents also tend to occur at intersections with stop signs, but it was found that the accident rate was twice as high as that at intersections without stop sign.



Fig.16 Accident rate by stop sign (by party)

Therefore, we considered about the cause of accidents at intersections with stop sign. The road where the stop sign is installed is an intersection where there is a risk of traffic accidents such as curve, gradients, road works, etc. where the left and right sides cannot be seen ${ }^{[23]}$. According to the results of analysis of the relationship between the prospect of an intersection and the occurrence of a crossing collision accident, it was found that there were a lot of accidents where the crossing accident had poor visibility ${ }^{[24]}$. Based on this conclusion, the reason why both car to car and car to bicycle accidents have high accident rates at intersections with stop sign is that there 
are many intersections with poor visibility and with stop sign in road planning, and failure to confirm safety may also lead to accidents.

\section{3-4-2. By stop line setback}

This part clarified the characteristics of the accident by party, by accident rate comparison of whether there was a stop line setback at the intersection. This part is an indepth research of 3-4-1. Therefore, the setback of the stop line was investigated by examining the setback of the 1,610 intersections with stop sign.

The target 1,610 intersection are classified according to the presence or absence of setback of the intersection stop line. The criterion for the presence of Setback is the condition that the distance between the front end of the stop line and the intersection road boundary line is $\geq 1 \mathrm{~m}$. The distance measurement method is Google map. Calculated by scale conversion using satellite image function. It is classified into three types: no setback, one side setback, both setbacks. No setback means the distance between the front end of the stop line and the intersection road boundary line $\leq 1 \mathrm{~m}$ or an intersection where the stop line is not present. One side setback is an intersection where the stop line is retracting only on one side of the priority road. Both setbacks are an intersection where the stop lines on both sides of the priority road are present.

According to the presence or absence of these stop line setbacks, the distribution of the number of intersections is showed in Fig.17. Out of 1,610 intersections, there are 920 no setback intersections, accounting for more than half. The number of intersections with both setbacks is the least.

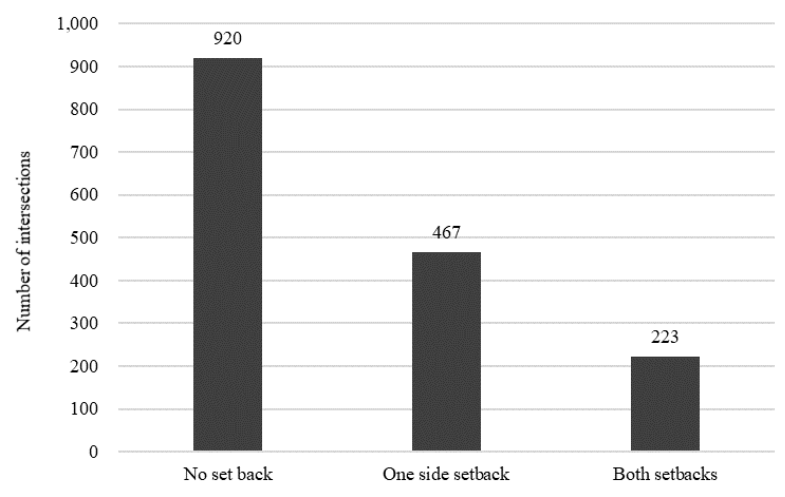

Fig. 17 Number of intersections by stop line setback

Fig.18 shows the distribution of the number of accidents that occurred at the intersections of the above three types of stop line setbacks by party. Both car to car accident and car to bicycle accidents occur at the intersections of stop line no setback. However, the number of one side car to car accidents occurred at intersections with one side setback and both setbacks is almost the same, car to bicycle accidents at the one side setback intersections are obviously more common than car to bicycle accidents at the both setbacks intersections.



Fig.18 Number of accidents by stop line setback (by party)

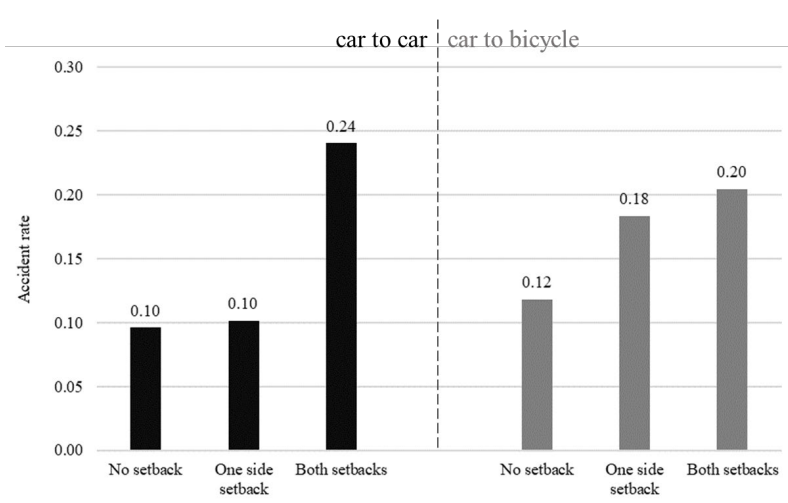

Fig.19 Accident rate by stop line setback (by party)

By calculating the accident rate, the distribution of accident frequency by party is showed in Fig.19. Comparing the characteristics of each party, car to car accidents tend to occur only when both stop lines have setbacks. On the other hand, it was found that car to bicycle accidents are likely to occur no matter whether the stop lines has one side setback or both setbacks.

From this result, we can see that the biggest difference between car to car and car to bicycle accidents is the one side setback part. However, after investigation, it was found that amongst the 467 intersections on one side setback, 320 intersections (about 68.5\%) were T-shape roads. We can see from Fig. 6 above that the accident rate of car to car on the T-shape road is $60 \%$ lower than that of car to bicycle. Perhaps the biggest factor affecting the accident here is not the setback of the stop line, but the shape of the intersection. Therefore, it is extremely important to propose the $\mathrm{T}$-shape road separately and perform the setback analysis of the stop line.

Therefore, below we have separately extracted the Tshape road for analysis. Since T-shape roads have crossing roads on only one side of priority roads, there is no both side setbacks' classification of the stop line. Therefore, there are no setback and one side setback when classified by the presence or absence of stop line setback. The number of T-shape roads by stop line setback is shown in Fig.20. Accidents that occurred at these 
intersections are summarized by party and shown in Fig.21. Furthermore, when the accident rate is calculated using the accident rate formula, the distribution of accident frequency by party is shown in Fig. 22.

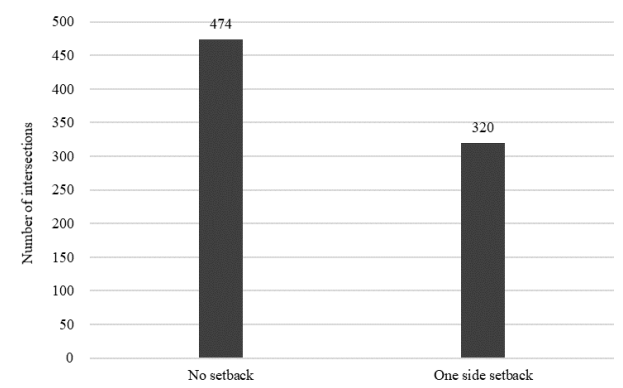

Fig.20 Number of T-shape roads by stop line setback



Fig.21 Number of T-shape roads by stop line setback (by party)



Fig.22 Accident rate of T-shape roads by stop line setback

What we can see here is that whether car to car accidents have a setback of the stop line, the frequency of occurrence does not change significantly. On the other hand, it was found that the car to bicycle accident is very likely to occur when there is a setback of the stop line on $\mathrm{T}$-shape roads.

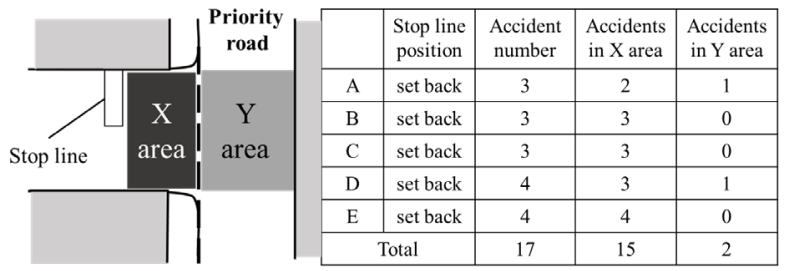

Fig.23 Investigation of car to bicycle accident-prone intersections
Therefore, in order to find the reason for the high accident rate of car to bicycle caused by the stop line setback, we investigated the T-shaped intersection where 3 or more car to bicycle accidents occurred within 3 years in Hakata City and conducted an analysis. As shown in Figure 23, we have defined the location at the time of the accident. $X$ area refers to the area between the stop line and the priority road' s borderline, and $\mathrm{Y}$ area refers to the intersection of two roads existing on the priority road area. We can see from the table on the right that all stop lines at the 5 intersections are set back, and accidents that occur in $\mathrm{X}$ area account for $88 \%$ of all accidents. From this, we can infer that the cause of the accident was that the car that entered the priority road from the non-priority road stopped near the stop line without sufficient safety check, or the car has not fulfilled its obligation to stop before the stop line, resulting in negligence of the bicycle that suddenly appeared from the blind spot.

\section{Conclusions}

In this paper, we selected Fukuoka prefecture for the research and conducted a crossing collision accident characteristics' analysis. In order to be able to find different characteristics caused by different accident parties, as to implement countermeasures separately. We classified crossing collision accidents into car to car and car to bicycle accidents and compared the frequency of accidents (accident rate) through various environmental factors existing at intersections, and the characteristics of each are clarified as follows.

The car to car crossing collision accident is more likely to occur at crossroads. When there are direction restrictions on some crossing roads at an intersection, the accident rate of car to car accident tends to be about $30 \%$ less because of the width of roads. It is also 3 times more likely to occur at intersections with stop signs than without stop signs and tend to occur only when both stop lines are setback.

On the other hand, the car to bicycle crossing collision accident tends to occur due to increase in the number of intersection roads. It is 2 times more likely to occur at intersections with stop signs than without stop sign and tends to occur if the stop line setback. Car to bicycle accidents are most likely to occur especially when there is a stop line setback on T-shape roads.

Based on the above-explained conclusions, the causes of the accident are explained by us as follows.

1) At T-shape intersections where the stop line is setback, insufficient safety check or no temporary stop before the stop line by car may cause a collision with a bicycle.

2) Bicycle's inability to recognize the traffic signs and ignore traffic signs due to no bicycle license required in Japan may be one of the reasons of the crossing collision accident. 


\section{Acknowledgments}

In this paper, the survey of the intersections, we received the cooperation, such as the provision of accident statistics data from Fukuoka Prefectural Police Traffic Planning Division. we received assistance from Rohit Shiv Prasad for English translation who is a student at our laboratory. I would like to express my gratitude.

\section{References}

[1] National Police Agency, "Traffic accident statistics", www.npa.go.jp/publications/statistics/koutsuu/toukeihyo. html , 2018

[2] Institute for traffic accident research and data analysis(ITARDA), "Basics of traffic accident statistics 2017”, www.itarda.or.jp/service/term, 2017

[3] National Police Agency Transportation Bureau, "Occurrence of traffic accidents during 2018", pg23, 2019

[4] Central Traffic Safety Measures Conference, "Traffic Safety Basic Plan: Towards a Society without Traffic Accidents", 2016

[5] ZMP, "Basic information on ADAS development", https://www.zmp.co.jp/knowledge/adas dev

[6] Institute for traffic accident research and data analysis (ITARDA), "Traffic accident statistics glossary". www.itarda.or.jp, 2017

[7] Sato, J., Hirota, M., \& Nomura, T., "Analysis of intersection environment to prevent crossing collision accidents", 16th ITS Symposium - Doshisha University, Kyoto, 2018

[8] Sato, J., "Study on prevention of crossing collision accidents based on intersection environmental analysis", Kyushu University Master Thesis, 2019

[9] Nomura, T., Hirota, M., \& Sato, J., "Mutual effect analysis of intersection environment to prevent crossing collision accident", 17th ITS Symposium, 2019

[10] Nomura, T., "Study on Factors of Occurrence of Encounter and Accident Prevention", Kyushu University Master Thesis, 2020

[11] Institute for traffic accident research and data analysis (ITARDA), "Crossing collision accident seen from road environment". www.itarda.or.jp, 2007

[12] Institute for traffic accident research and data analysis (ITARDA), "Special feature: Analysis of human factors in Crossing collision accidents", 2005

[13] Kenji Hagita \& etc., "Analysis of bicycle accident focusing on traffic direction", Japan Society of Civil Engineers, Vol.69, No.5, 1_781-1_788, 2013

[14] Kenji Hagita \& etc., "Analysis of intersection bicycle accidents focusing on the direction of travel of bicycles", Japan Society of Civil Engineers, Vol.70, No.5, 1 1023$1 \_1030,2014$

[15] Kenji Hagita \& etc., "Comparison of accidents involving roadside bicycles in Shizuoka prefecture by traffic direction”, Japan Society of Civil Engineers, Vol.75, No.5, 1 989-1 998, 2019

[16] Suzuki Mio \& etc., "Bicycle accident analysis on roads with sidewalks in urban areas", Japan Society of Civil Engineers, Vol.69, No.5, 1_715-1_724, 2013

[17] Suzuki Mio \& etc., "Bicycle accident analysis on roads without sidewalks in urban areas", Japan Society of Civil Engineers, Vol.70, No.5, 1_771-1_779, 2014
[18] Institute for traffic accident research and data analysis(ITARDA), "Traffic accident annual report", https://www.itarda.or.jp/materials/traffic_accident/free, 2018

[19] Fukuoka Prefectural Police Headquarters Traffic Planning Division, "Heise 29nentyuno zinshinziko", https://ckan.open-governmentdata.org, 2018

[20] Fukuoka Prefectural Police, "April 2018 Fukuoka prefectural police provided Statistical information about crossing collision accident, 2018

[21] Google, "Google Map", https://www.google.co.jp/maps/

[22] Akio Takahashi, "Special Feature: Crossing collision accidents between bicycles and cars - Beware of bicycles jumping out at intersections with poor visibility", Japan Traffic Accident Analysis Center, 2017, No.122, 2017

[23] National Police Agency, "Traffic regulation standards",https://www.npa.go.jp/laws/notification/koutu u/kisei/kisei20170424.pdf , 2017

[24] Nomura, T., Hirota, M., \& Sato, J., "Evaluation of the Driver Visibility Affecting the Occurrence of Crossing Accidents”, 17th ITS Symposium, 2019

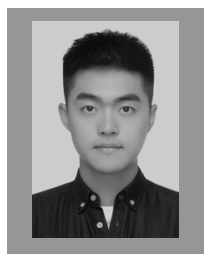

Jiaao Dong He was born at Fushun, Liaoning in China. He graduated Dalian Jiaotong University, China in 2018. Now he belongs to Graduates School of Kyushu University. He is a member of the JSAE.



Masaki Hirota He was born at Tokyo in 1962. He received the ME degree and Dr. Eng. Degree in 1987 add 2006, respectively. He is currently a professor of Kyushu University. He is a member of the IEEJ, JSAE, JITR and JSAP.

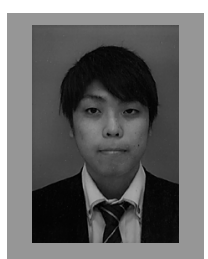

Takumi Nomura, He graduated Kumamoto University in 2018. He graduated the Department of Automotive Science Graduates School of Integrated Frontier Sciences, Kyushu University in 2020 . He is a member of the JSAE.

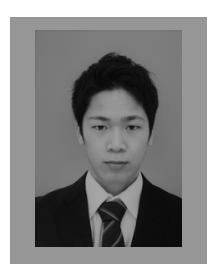

Junya Sato He graduated Department of Electrical Engineering and Computer Science, Kyushu University in 2017. He graduated the Department of Automotive Science Graduates School of Integrated Frontier Sciences, Kyushu University in 2019. He is a member of the JSAE. 